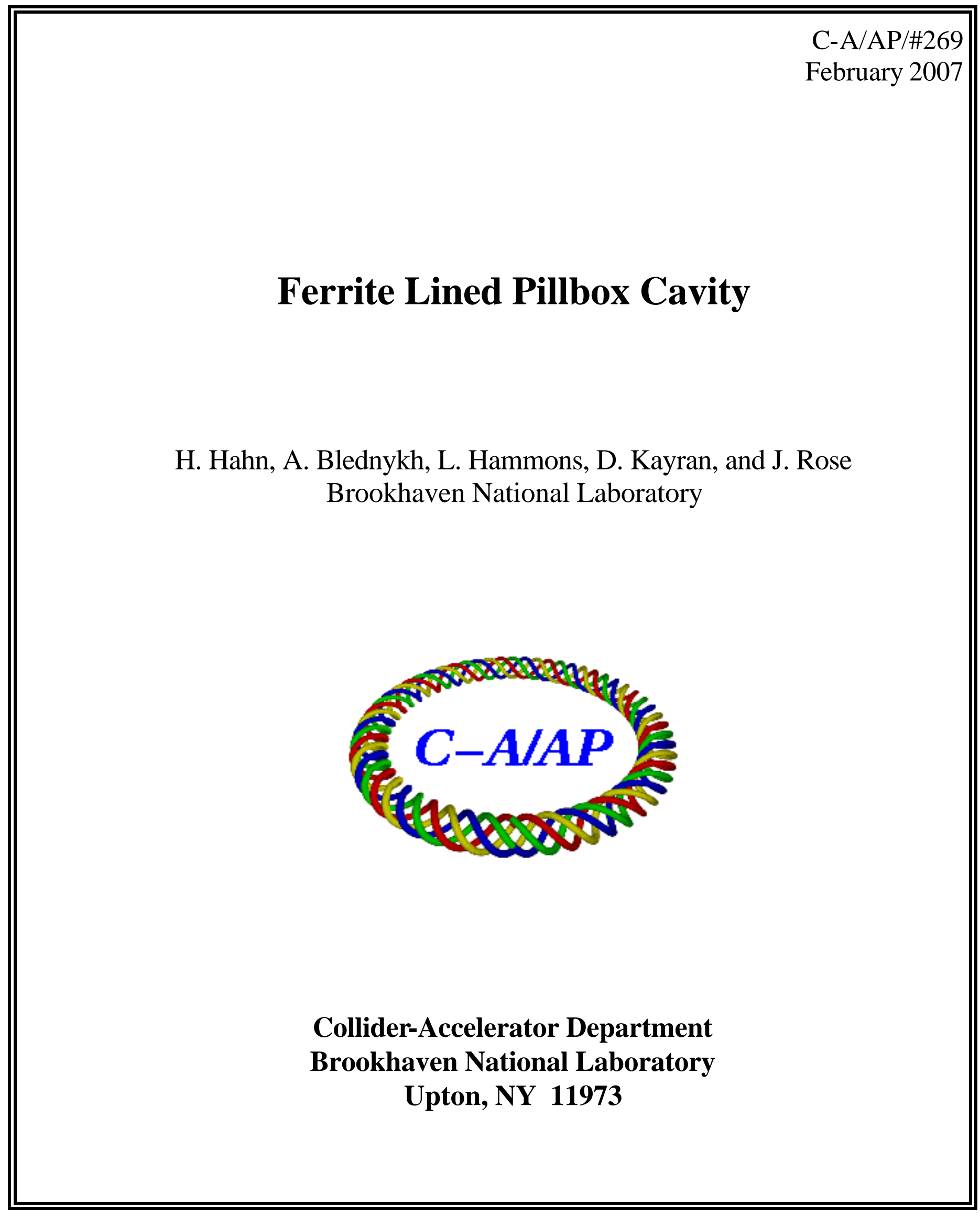




\title{
Ferrite Lined Pillbox Cavity
}

\author{
H. Hahn, A. Blednykh, L. Hammons, D. Kayran, and J. Rose \\ Brookhaven National Laboratory
}

\section{Introduction}

An Energy Recovery Linac (ERL) is being constructed at this laboratory to demonstrate feasibility of electron-cooling for RHIC [1]. Reducing higher order modes (HOM) in the superconducting accelerator cavity is one of several challenges and, following the techniques developed at KEKB [2] and Cornell [3, 4], will be addressed by means of a ferrite absorber at room temperature.

The HOM absorber for the ECX superconducting 5-cell cavity is a cylindrical spool with ferrite tiles attached to the wall, similar to a test model shown in Fig. 9. The operational unit has 18 plate sections in the $25 \mathrm{~cm}$ diameter spool, each attached with two tiles of $2 \times 1.5 \times 0.125$ in. dimensions. The model has been assembled from surplus ferrite tiles in the shape of a ferrite-lined pill box and serves for diverse measurements to determine its properties and its damping effect on resonances in the copper cavity prototype. A large quantity of experimental data was collected and preliminary results are reported elsewhere [5]. A full analysis of the data however requires the development of a frame work involving theoretical field analysis as well as the use of computer codes. This paper presents some relevant studies and attempts to explain the attenuation properties specific to ferrite losses. A rigorous analytical treatment of the absorber with its ferrite plates is practically precluded and is here replaced by an analysis of a ferrite lined circularly and longitudinally uniform wave structure. During operation, the absorber structure functions as a waveguide attenuator and its damping is expressed in terms of $\mathrm{dB} / \mathrm{m}$. However, a more convenient way of measuring the HOM damping properties consists in placing shorting plates at either end of the model thereby transforming it into a low-Q "pillbox" cavity [5]. Resonance frequencies and Q-values can then be obtained from S21 scattering coefficient measurements.

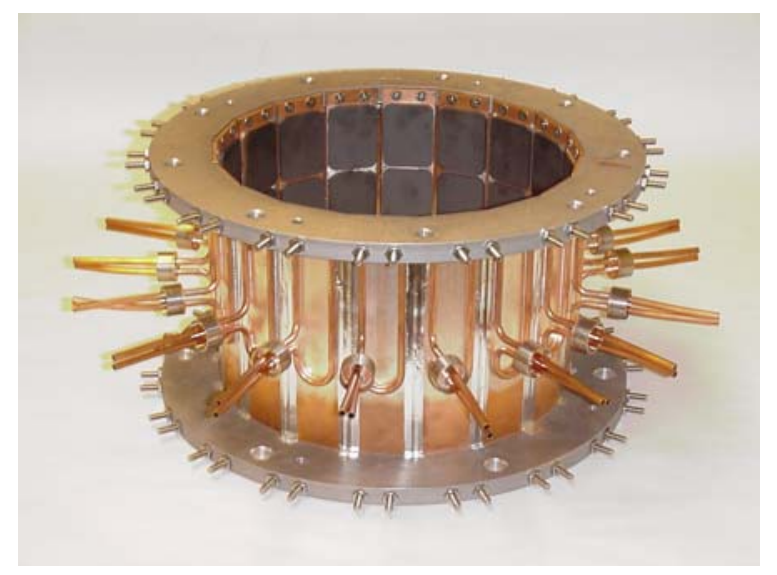

Figure 1. HOM ferrite absorber test unit 
In order to develop a better understanding of the ferrite absorber properties the pillbox was analyzed analytically using standard electro-magnetic field equations and run in electro-magnetic simulation programs based on complex permeability values. Vice versa, using the analytical or simulation method for the interpretation of the pillbox measurements yields the complex permeability for subsequent use in general structures. In addition to this more rigorous approach, the possibility of interpreting the absorber properties as a perturbation was also considered. Using a surface impedance (or R-square for losses alone) is a convenient way of interpreting measurements and could be directly applied in simulation programs to predict of HOM absorber performance

Resonances in a ferrite-lined cylindrical cavity can be analytically expressed in terms of individual modes with explicit expressions for the field components and the associated wave numbers. The ferrite material properties are defined for the analysis by complex, frequency dependent permeability and permittivity parameters. The fields must satisfy the tangential boundary condition on the metallic enclosure and the continuity condition at the interface between vacuum and ferrite and lead to the complex eigen-frequencies and decaying oscillations.

The lowest resonance of the pillbox shows a TM010 field distribution which is undistinguishable from a purely high loss metallic cavity. Of special interest, and the initial stimulus for this note, is the shape of the fields within the ferrite tiles of the absorber and any similarity to the fields due to the classical metallic skin effect. The ferrite tiles are thin compared to the cavity dimensions and their properties conceivably could be interpreted as a perturbation in analogy to the skin effect of lossy metals. Treating the fields in the ferrite as a skin effect points to a similarity of metallic conductivity and the imaginary part of the ferrite permeability as being responsible for the damping effect.

In the first section of this report, a rigorous analytical treatment of the electric fields in both, vacuum and ferrite, regions of the structure is derived and the conditions for attenuation of propagation in a waveguide or the resonance frequency and Q-value of a cavity are given. A simple generic model of the complex ferrite permeability is generated from available sources and it is used for a detailed skin effect analysis. Using typical permeability parameters, the attenuation constant in a lined wave guide and the eigen-frequency, Q-value and field shapes for the TM010 and TM020 mode in a ferrite lined pill box cavity are obtained from the rigorous analytical expressions via the Mathematica 5.2 program. The results are then compared with results from electromagnetic simulation programs, in particular the CST Micro Wave Studio (MWS) [6], the GdfidL [7], and Superfish program. Adequate agreement of analytical results and MWS simulations was found, but some differences with the other programs are noticed and are pursued by the respective experts

Somewhat beyond the objective for this report and more as illustration of the concepts presented here, the results from a S21 measurement of a short ferrite-lined cavity are summarily interpreted while relegating a detailed discussion to a future report. 


\section{Circular cylindrical geometry}

For the purpose of the theoretical analysis of wave propagation in the HOM ferrite absorber, the actual tiled shape is replaced by a circular ferrite cylinder in a lossless waveguide. The inner radius of the ferrite is $a$ and the outer radius $b$ is touching the circular waveguide. The time-harmonic fields in the ferrite-free inner region, with the time factor $e^{j \omega t}$ omitted, are given by

$$
\begin{gathered}
E_{z i}=\frac{k^{2}-k_{z}^{2}}{k_{r i} k} J_{0}\left(k_{r i} r\right) e^{-j k_{z} z} \\
E_{r i}=j \frac{k_{z}}{k} J_{1}\left(k_{r i} r\right) e^{-j k_{z} z} \\
Z_{0} H_{\Theta i}=j J_{1}\left(k_{r i} r\right) e^{-j k_{z} z}
\end{gathered}
$$

where the wave numbers are obviously $k=\omega / c, k_{z}$, and $k_{r i}$. Note that in order to simplify the numerical work, natural units are used while retaining the MKS convention regarding $4 \pi$ in the field equations, $\left(c=1, \mu_{0}=1, \varepsilon_{0}=1 Z_{0}=\sqrt{\mu_{0} / \varepsilon_{0}}=1\right)$

The field components in the ferrite have the same propagation constant, $e^{-j k_{z} z}$, and must satisfy the boundary condition $E_{z}(b)=0$, be continuous in $E_{z}$ and $H_{\Theta}$ at $r=a$, and can be written as

$$
\begin{aligned}
& E_{z o}=\frac{\varepsilon \mu k^{2}-k_{z}^{2}}{\varepsilon k_{r o} k} \frac{J_{1}\left(k_{r i} a\right)}{F_{1}\left(k_{r o} a\right)} F_{0}\left(k_{r o} r\right) e^{-k_{z} z} \\
& E_{r o}=j \frac{k_{z}}{\varepsilon k} \frac{J_{1}\left(k_{r i} a\right)}{F_{1}\left(k_{r o} a\right)} F_{1}\left(k_{r o} r\right) e^{-k_{z} z} \\
& Z_{0} H_{\Theta o}=j \frac{J_{1}\left(k_{r i} a\right)}{F_{1}\left(k_{r o} a\right)} F_{1}\left(k_{r o} r\right) e^{-k_{z} z}
\end{aligned}
$$

with the relative material parameters $\varepsilon \approx \varepsilon^{\prime}, \mu=\mu^{\prime}-j \mu^{\prime \prime}$, and the Bessel-function combinations

$$
\begin{aligned}
& F_{0}\left(k_{r o} r\right)=J_{0}\left(k_{r o} r\right) Y_{0}\left(k_{r o} b\right)-J_{0}\left(k_{r o} b\right) Y_{0}\left(k_{r o} r\right) \\
& F_{1}\left(k_{r o} r\right)=J_{1}\left(k_{r o} r\right) Y_{0}\left(k_{r o} b\right)-J_{0}\left(k_{r o} b\right) Y_{1}\left(k_{r o} r\right)
\end{aligned}
$$

Finding a solution involves satisfying three simultaneous conditions. Vanishing divergence, $\nabla E=0$, provides two conditions on the wave numbers,

$$
k^{2}-k_{r i}^{2}-k_{z}^{2}=0 \text { and } \varepsilon \mu k^{2}-k_{r o}^{2}-k_{z}^{2}=0
$$

and continuity of $E_{z}$ at $r=a$ yielding the necessary third ( $H_{\Theta}$ at $r=a$ is built-in),

$$
\frac{k^{2}-k_{z}^{2}}{k_{r i} k} J_{0}\left(k_{r i} a\right)=\frac{\varepsilon \mu k^{2}-k_{z}^{2}}{\varepsilon k_{r o} k} \frac{J_{1}\left(k_{r i} a\right)}{F_{1}\left(k_{r o} a\right)} F_{0}\left(k_{r o} a\right)
$$

The solution is obtained after simplification from the two necessary simultaneous equations in $k_{r i}$ and $k_{r o}$,

$$
\varepsilon k_{r i} J_{0}\left(k_{r i} a\right) F_{1}\left(k_{r o} a\right)-k_{r o} J_{1}\left(k_{r i} a\right) F_{0}\left(k_{r o} a\right)=0
$$




$$
(\varepsilon \mu-1) k^{2}+k_{r i}^{2}-k_{r o}^{2}=0 .
$$

Wave propagation, $e^{-j k_{z} z}$, in a ferrite lined guide follows from $k_{z}^{2}=k^{2}-k_{r i}^{2}$. Cavity resonances are found by imposing appropriate values for $k_{z}$, such as $k_{z}=0$ for the TM 010 and TM020 modes yielding $k=k_{r i}$. The eigen value solution provides the observable resonance frequency and the quality factor from

$$
\begin{gathered}
f_{O}=\frac{c}{2 \pi} \operatorname{Re} k_{r i} \\
Q=\frac{\operatorname{Re} k_{r i}}{2 \operatorname{Im} k_{r i}}
\end{gathered}
$$

\section{The Ferrite Model}

One major objective for this study is a comparison of the magnetic field penetration into the ferrite and the concomitant energy losses. The exact analytical expressions presented above are limited to rotationally symmetric structures. In contrast, the actual HOM absorber is constructed from a sequence of flat tiles and locally can be handled in a Cartesian coordinate system in analogy to the classical skin effect for metals. A further uncertainty, not yet studied, derives from the gaps between the tiles in the actual device. All geometric irregularities can in practice best be addressed with simulation programs which however also have limitations as to accuracy. Common to any theoretical approach is the need for accurate ferrite permeability data. For the mostly qualitative discussion of ferrite absorber properties in this paper, a generic complex permeability model is needed and adequate.

For simplicity's sake, the ferrite is considered homogenous and isotropic and the frequency dependence is either neglected or given as first-order Debye model. One can distinguish the typical material properties as follows for

$$
\begin{array}{ll}
\text { conducting metal }(\mathrm{Cu}): & \sigma \sim 6.4 \times 10^{7} / \Omega \mathrm{m}, \mu^{\prime} \sim 1, \mu^{\prime \prime} \sim 0, \sigma \gg \varepsilon^{\prime} \omega \text {, and } \\
\text { ferrite: } & \sigma \sim\left(10^{-5} \div 10^{-7}\right) / \Omega \mathrm{m}, \sigma \ll \varepsilon^{\prime} \omega @ f>1 \mathrm{MHz},
\end{array}
$$

Ferrite permeability properties, shown in Fig. 2, can be taken from Hartung's thesis and publications, as $\mu^{\prime} \approx 2.2$ and $\mu^{\prime \prime} \approx 12$ at $\sim 1 \mathrm{GHz}, \varepsilon^{\prime} \approx 13$ and $\sigma \approx 10^{-5} / \Omega$.m. [8] In the $\mathrm{GHz}$ region of interest to the HOM absorber the electric conductivity is negligible and the permeability, again in natural units with, can be presented with sufficient accuracy by a complex model, shown in Fig. 3, again in natural units with $c=1, \mu_{0}=1, \varepsilon_{0}=1$,

$$
\mu=\mu_{0}\left\{\mu^{\prime}-j \mu^{\prime \prime}\right\} \approx \frac{70}{1+j 5.5 f_{G H z}},
$$

The ERL HOM absorber uses nickel-zinc ferrite C-48, produced by Countis Industries, for which the permeability properties are only known from the small sample measurements by Mouris and Hutcheon [9]. The measured permeability of the CLS data at room temperature is shown in Fig. 4 , and can be modeled from the value $\mu \approx 4-\mathrm{j} 10$ at $1 \mathrm{GHz}$ yielding the model representation also shown in Fig. 4. 


$$
\mu \approx \frac{30}{1+j 2.5 f_{G H z}}
$$

It is to be noted that $\mu^{\prime}$ is well represented by the model but the $\mu^{\prime \prime}$ value is overestimated at higher frequencies
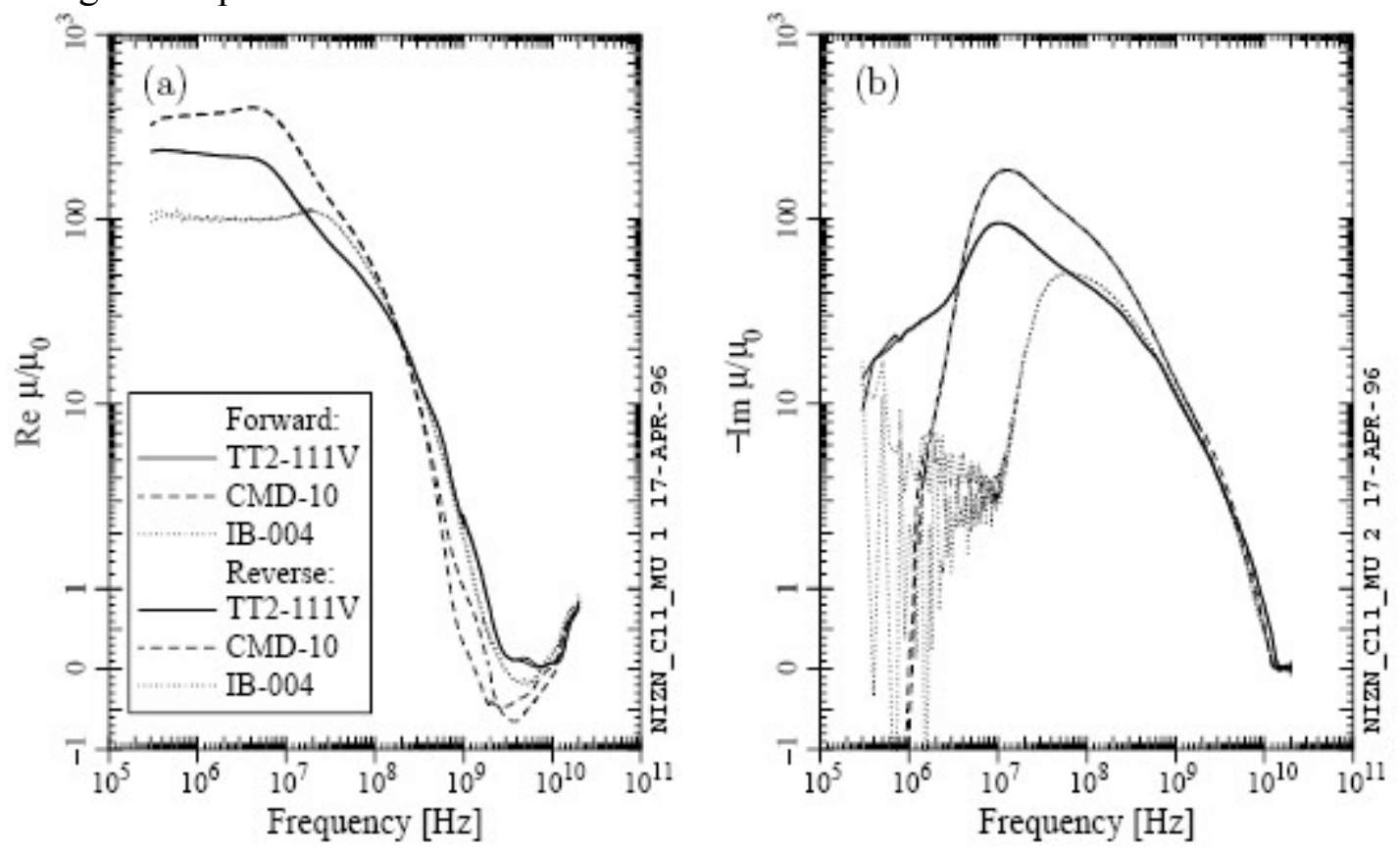

Figure 2. Ferrite material properties from Hartung [8] .

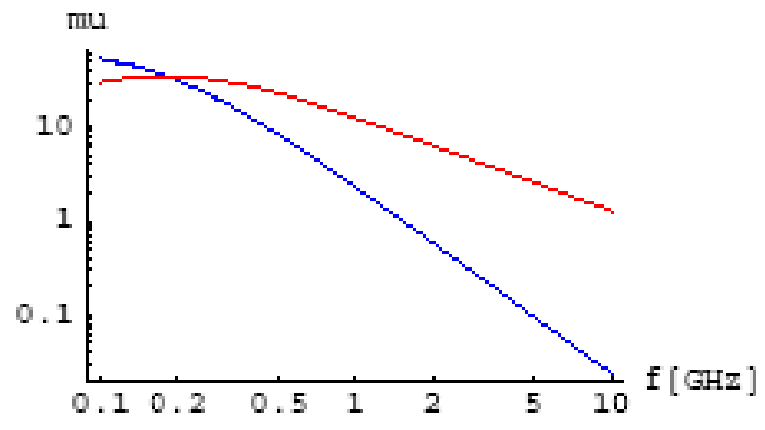

Figure 3 .Complex Ferrite model for skin depth simulations

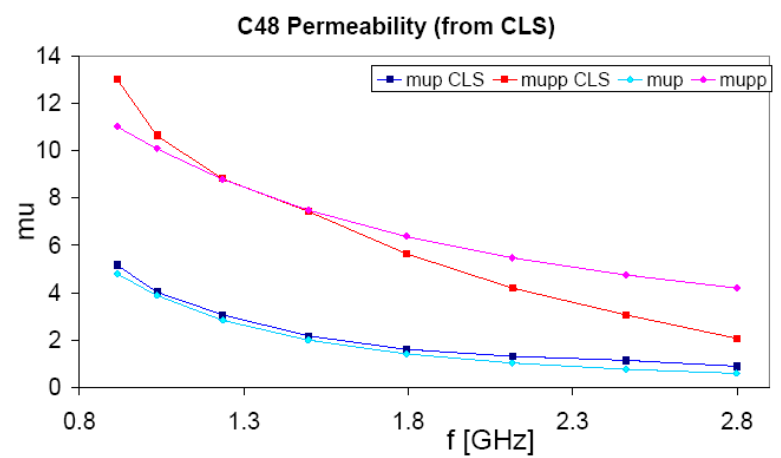

Figure 4. Complex Ferrite models for skin depth simulations 
In addition to the frequency dependence, the ferrite permeability and its HOM damping properties change with temperature. Higher operational temperature reduces the absorber capability [9] and cooling is essential. Operation at liquid nitrogen temperature was studied at Cornell and led to the choice of different materials depending on the frequency range [10]. In fact, the ongoing absorber study and the present note are done to establish the ferrite properties in the in-situ configuration of the BNL ERL HOM absorber.

\section{Skin effect analysis}

The ferrites are flat tiles whose dimensions are very small compared with the beam tube radius so that the e. $\mathrm{m}$. fields in the ferrite can be analyzed in a Cartesian coordinate system in full analogy to the "classical" skin effect treatment. The fields in the material are locally driven by a magnetic field $H_{y}(z=0) \omega^{j \omega t}$ which is due to an incoming TEM wave perpendicular to the $x-y$ surface and position independent over the inner surface. The time-harmonic electric field in the ferrite material is derived from the solution of the wave equation

$$
\nabla^{2} E+\mu(\varepsilon-j \sigma / \omega) \omega^{2} E=0
$$

Taking into account the boundary condition $E_{x}(z=\tau)=0$ to a perfect conductor at the outer ferrite wall, the field expressions are found as follows

$$
\begin{aligned}
& E_{z}=-\frac{k_{x}}{\varepsilon k} \cosh \kappa(\tau-z) e^{-j k_{x} x} \\
& E_{x}=-j \frac{\kappa}{\varepsilon k} \sinh \kappa(\tau-z) e^{-j k_{x} x} \\
& Z_{0} H_{y}=\cosh \kappa(\tau-z) e^{-j k_{x} x} .
\end{aligned}
$$

Zero divergence yields the condition on the wave number

$$
\kappa^{2}=2 k_{x}^{2}-\mu \varepsilon_{0}\left(\varepsilon^{\prime}-j\left(\varepsilon^{\prime \prime}+S\right)\right) k^{2}
$$

with $\mu \simeq \mu_{0}\left(\mu^{\prime}-j \mu^{\prime \prime}\right), \varepsilon=\varepsilon_{0}\left(\varepsilon^{\prime}-j \varepsilon^{\prime \prime}\right)$, and $S=\sigma / \varepsilon_{0} \omega$. The assumption of a position independent driving magnetic field in the $\mathrm{x}-\mathrm{y}$ plane, implies $k_{x} \approx 0$. The approximation $\kappa^{2} \approx-\mu \varepsilon k^{2}$ is valid for any material leading to the expression for the surface impedance in the $\mathrm{x}-\mathrm{y}$ plane

$$
\mathfrak{R}=-j Z_{0} \frac{\kappa}{\varepsilon k} \tanh \kappa \tau .
$$

Although generally valid, both for the high-conductivity metals as well as the ferrite parameters considered here, it is instructive to consider them separately. The skin effect in metal is reviewed first. The wall thickness of metal is usually negligible so that $\tanh \kappa \tau \approx 1$ and the conductivity in metals dominates permittivity, $S \gg \varepsilon^{\prime}, \varepsilon^{\prime \prime}$, and $\mu^{\prime} \gg \mu^{\prime \prime}$, leading to

$$
\mathfrak{R}_{\infty} \approx Z_{0} \sqrt{j \mu^{\prime} / S}=(1+j) Z_{0} \sqrt{\mu^{\prime} \omega / 2 \sigma},
$$

and the skin depth 


$$
\delta=\frac{1}{\operatorname{Re} \kappa} \approx \frac{1}{k} \sqrt{\frac{2}{\mu^{\prime} S}}=\sqrt{\frac{2}{\mu \omega \sigma}}
$$

in full agreement with the well-known "classical" results.

\section{Skin effect in flat ferrite plates}

The material parameters of ferrite differ qualitatively from a metal, notably due to $\sigma \sim 0$, and require a separate discussion. However, in the frequency range of interest, the material is in good approximation determined by generic ferrite properties with only $\mu^{\prime}, \mu^{\prime \prime}, \varepsilon^{\prime}$. The skin effect formulas collected above together with the generic permeability model, $\mu \approx 30 /\left(1+j 2.5 f_{G H z}\right)$, shown in Fig. 3 provide the relevant numerical results for the flat ferrite plates. The complex surface impedance of a ferrite tile with finite thickness, $\tau$, and attached to a perfect conductor is given by

$$
\mathfrak{R}=Z_{0} \sqrt{\frac{\mu^{\prime}-j \mu^{\prime \prime}}{\varepsilon^{\prime}}} \tanh \left(k \tau \sqrt{-\left(\mu^{\prime}-j \mu^{\prime \prime}\right) \varepsilon^{\prime}}\right)
$$

and the wave number by

$$
\kappa=k \sqrt{-\left(\mu^{\prime}-j \mu^{\prime \prime}\right) \varepsilon^{\prime}}
$$

Numerical values for the wave number in the infinite ferrite are shown in Fig. 4 over a broad frequency range. However, in the $\mathrm{GHz}$ region relevant to the present study, $\mu^{\prime \prime} \geq \mu^{\prime}$, and $\kappa$ can be obtained from the "asymptotic" expression $\kappa_{\infty} \sim k \sqrt{j \mu^{\prime \prime} \varepsilon^{\prime}}$

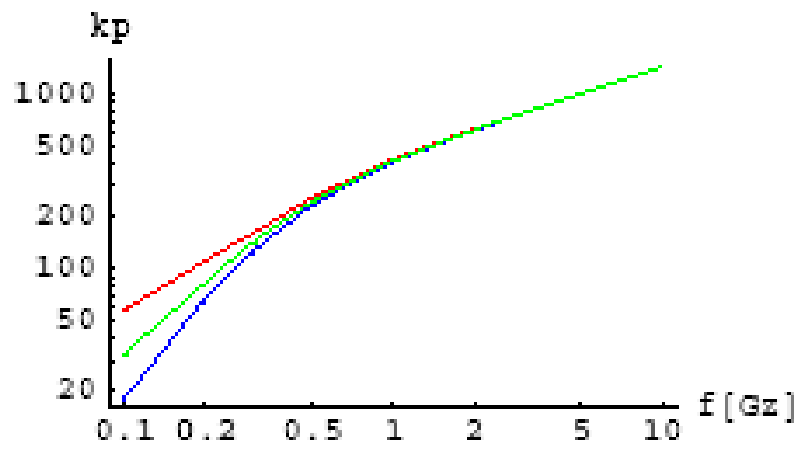

Figure 5. Real (blue) and imaginary (red) component of the wave number, $\kappa \equiv k p$, versus frequency $(\mathrm{GHz})$. The green curve is from the asymptotic approximation.

The nominal skin depth, defining the field penetration in the infinite ferrite, is shown in Fig. 6 by the blue curve together with the $3 \mathrm{~mm}$ limit due to the tile thickness. Note that the skin depth is about equal to the tile thickness at frequencies at $\sim 0.5 \mathrm{GHz}$, but that in order to neglect the wall thickness, one must have $\delta \ll \tau$. Furthermore, the graph shows that in the frequency range of interest, $f \geq 0.5 \mathrm{GHz}$, the skin depth is given by the asymptotic order-of -magnitude estimate as

$$
\delta_{\infty}=\frac{1}{\operatorname{Re} \kappa} \sim \frac{1}{k} \sqrt{\frac{2}{\varepsilon^{\prime} \mu^{\prime \prime}}}
$$




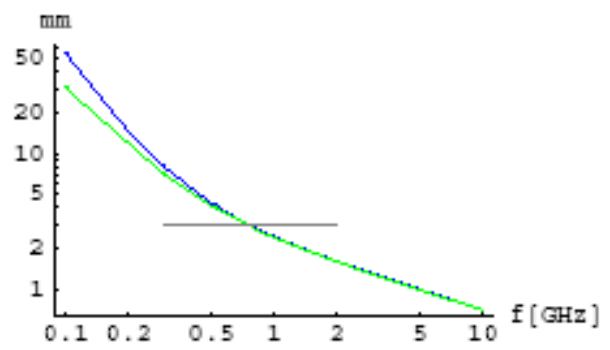

Figure 6. "Skin depth" (asymptotic green) in infinite model ferrite vs frequency (GHz)

A finite wall thickness modifies the magnetic field penetration and changes the surface impedance by the $\tanh \kappa \tau$ factor. Taking the wall thickness as $\tau=3 \mathrm{~mm}$, one finds the correction factor in Fig. 7. It is seen that a finite wall thickness has a significant impact in the low $\mathrm{GHz}$ region but that well above $1 \mathrm{GHz}, \tanh \kappa \tau \sim 1$, and it can be neglected for a qualitative discussion.

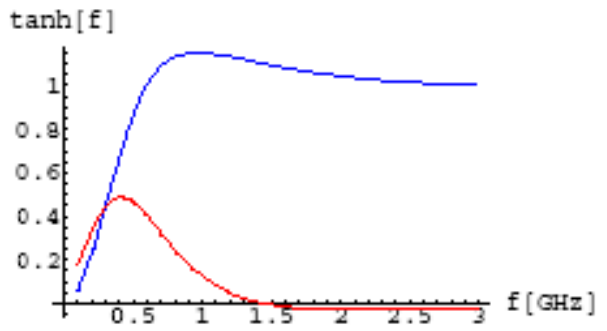

Figure 7. The correction factor $\tanh \kappa \tau$ versus frequency, blue is real and red the imaginary component.

The present paper was stimulated by the observation that in computer simulations the magnetic field shape differed from the expected uniform decay into the ferrite wall. In fact, the magnetic field, $H_{y}$ in the finite thickness ferrite is given by

$$
H_{y}=\frac{\cosh \kappa(\tau-z)}{\cosh \kappa \tau}
$$

and only in the unbound wall, if $\kappa \tau \gg 1$, by the expected $H_{y} \sim \exp (-\kappa z)$. The Fig. 8 shows the absolute value of the magnetic field penetrating into the ferrite for a wall thickness of $\tau=5$ (black), 50 (red), and the unbound (green) case. The solid colors curves are at the TM010 resonance with $\mu^{\prime \prime}=5$ and the dashed at the TM020 resonance with $\mu^{\prime \prime}=2$. The graphs show that the magnetic field penetrates fully into the ferrite plates in contrast to the usual situation in metal.

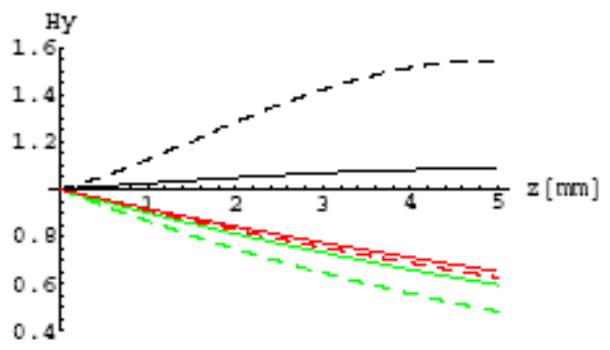

Figure 8. Magnetic field strength in the ferrite plate. 
The complex surface impedance of a ferrite tile with finite thickness, $\tau$, and attached to a perfect conductor is given by the above "full" expression. The real part of the surface impedance, here also called the R-square $\left(R_{S Q}=\operatorname{Re} \mathfrak{R}\right)$, is responsible for the losses and the cavity quality factor whereas the imaginary part causes a frequency shift of the cavity resonance. Using the generic model parameters, the real and imaginary components of the surface impedance for the model ferrite with $3 \mathrm{~mm}$ thick tiles are computed and shown in Fig. 7 together with the "asymptotic" values in which $\mu^{\prime \prime} \geq \mu^{\prime}$ and the tile thickness is neglected. One sees that in the high $\mathrm{GHz}$ region the real and imaginary impedance have essentially equal values, but that in the low $\mathrm{GHz}$ region, important to the HOM absorber properties, the full expressions are required.

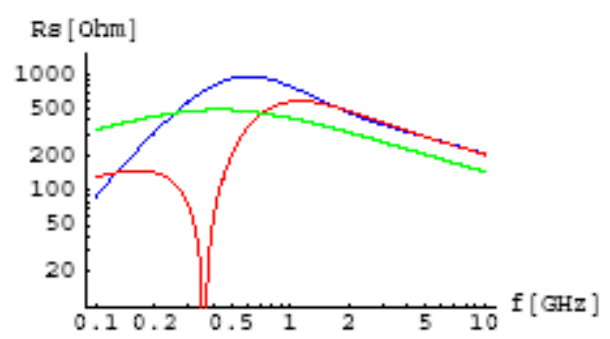

Figure 9. Real (blue) and imaginary (red) versus asymptotic (green) surface impedance

An intuitive understanding is gained from the asymptotic case of "high" frequencies and "thick" plates. At frequencies in the upper GHz region the generic permeability models show $\mu^{\prime \prime} \geq \mu^{\prime}$ and in the infinite thickness case one has $\tanh \kappa \tau \sim 1$. The "asymptotic" expressions for the surface impedance and skin depth become

$$
\begin{aligned}
& \Re_{\sim} \approx Z_{0} \sqrt{\frac{\mu^{\prime \prime}}{j \varepsilon^{\prime}}}=(1-j) Z_{0} \sqrt{\frac{\mu^{\prime \prime}}{2 \varepsilon^{\prime}}} \\
& \delta_{\sim} \approx 1 / \operatorname{Re} \kappa=\frac{1}{k} \sqrt{\frac{2}{\varepsilon^{\prime} \mu^{\prime \prime}}}
\end{aligned}
$$

Here $\mu^{\prime \prime} \& \varepsilon^{\prime}$ replace the $\sigma \& \mu^{\prime}$ in metals. A frequency dependence of the surface impedance is hidden in the variation of $\mu^{\prime \prime}$. In view of the approximate frequency independence of $\varepsilon^{\prime}$ and the inverse dependence of $\mu^{\prime \prime}$ in the GHz region, one finds the same frequency dependence in ferrite and metal. As a consequence, the ferrite losses increases with the square root of the imaginary permeability component, but decrease with frequency, and thus limit the usefulness of ferrite HOM absorbers at the highest frequencies [8].

\section{Ferrite lined Waveguide}

The HOM absorber for the ECX superconducting 5-cell cavity is a cylindrical spool with ferrites, similar to its test model shown in Fig.1. The unit has 18 sections in the $25 \mathrm{~cm}$ diameter spool, each assembled of two tiles with $2 \times 1.5 \times 0.125$ in. dimensions. A rigorous analytical treatment of its damping properties are practically precluded and are replaced by an analysis of a circularly symmetric waveguide structure with outer radius $b=12.4 \mathrm{~cm}$ and inner radius $a=11.9 \mathrm{~cm}$. (Note that a ferrite thickness $\tau=5 \mathrm{~mm}$ is used 
here and in subsequent simulation computations to limit the number of mesh points in the ferrite).

The absorber is attached to either end of the ERL cavity and its damping effect can be seen as a lossy waveguide or a lossy cavity. In this section, the waveguide properties are discussed. Axial wave propagation in the infinitely long ferrite lined guide is given by $e^{-j k_{z} z}$ or $e^{(\alpha-j \beta) z}$ in the standard notation. The attenuation coefficient $\alpha=\operatorname{Re} \kappa$ is obtained from the analytical expressions above and shown in Fig. 10 for the TM01 mode with a ferrite having a frequency independent $\varepsilon=12, \mu^{\prime}=2$ and $\mu^{\prime \prime}=0,2$, or 5 . The figure shows the attenuation coefficient, multiplied by the ferrite length of $\ell_{F e}=2 \times 5.08 \mathrm{~cm}$, as function of frequency normalized to the cutoff frequency of the empty and lossless cylinder with $f_{c o}=918.6 \mathrm{MHz}$, with $k_{c o}=19.24 / \mathrm{m}$, and $\lambda_{c o}=32.66 \mathrm{~cm}$. Also shown is the perturbative factor for a waveguide with wall losses from $\mu=2-\mathrm{j} 5$ [11]

$$
\alpha \ell_{F e}=\frac{R_{S Q}}{Z_{0}} \frac{\ell_{F e}}{b}
$$

The graph shows that the absorber is expected to attenuate a wave by not more than about $10 \mathrm{~dB}$ ( or $\alpha \ell_{F e} \leq 1.15$ ). Furthermore, the simple perturbative factor gives reasonable numerical results and represents qualitative estimates as guide for design choices.

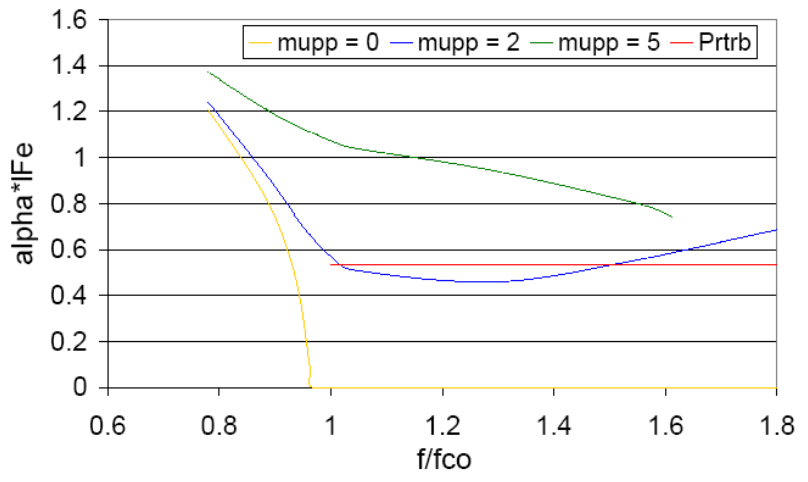

Figure 10. Waveguide attenuation

\section{Ferrite lined Cavity}

Determining the absorber losses in a waveguide configuration is challenging and its conversion into a cavity is preferable and allows the use of simpler network analyzer measurements. The cavity geometry is defined as a simple pill box with the metallic spool inner diameter of $24.8 \mathrm{~cm}$ encapsulating the $6.5 \mathrm{~cm}$ long and $5 \mathrm{~mm}$ thick ferrite ring. The spool was terminated with shorting end plates and excited with axially placed probes for scattering coefficient measurements reported elsewhere. The primary objective of the present study is to provide a basis from analysis and computer simulation for the interpretation of experimental data. Note that the length of the pill box is shorter than the HOM absorber in order to reduce the number of resonances. The excitable resonance frequencies of the empty, ferrite-free cavity are in the TM010 and TM020 modes at 0.9186 and $2.109 \mathrm{GHz}$ respectively, independent of the cavity length. 
Computer results for the ferrite lined cavity are obtained for these two resonances via the above analytical expressions with the Mathematica 5.2 (m52) program. The resonances are lowered by the insertion of a lossless ferrite with $\varepsilon^{\prime}=12, \sigma=0$, and $\mu^{\prime}=2$ to $883.7 \mathrm{MHz}$ and $1.975 \mathrm{GHz}$. Adding losses via the complex permeability, $\mu^{\prime \prime}$, while keeping the other parameters constant leads to the frequencies and quality factors in the Table I..

The interpretation of measurements as a perturbation assumes that the quality factor is determined by the ferrite surface impedance. It follows that the Q is obtained from the geometry factor of the lossless cavity together with the real part of the surface impedance (or $R_{S Q}=\operatorname{Re} \mathfrak{R}$ )

$$
Q \approx G / R_{S Q}
$$

The geometry factor of the TM0n0 modes in the ferrite lined pill box with perfect cylinder wall end caps is given by

$$
G_{0 n 0}=\frac{j_{0 n}}{2} Z_{0}
$$

where $j_{0 n}$ is the n-th solution of $J_{0}\left(j_{0 n}\right)=0$, leading to $G_{010} \approx 1.2024 \cdot Z_{0}$ and $G_{020} \approx 2.7604 \cdot Z_{0}$ respectively for the TM010 and TM020 resonances. The quality factors due to the ferrite perturbation, obtained from the full expression for the surface impedance, are also listed in Table I. At best, one finds an order of magnitude agreement for the quality factor from the perturbation and analytical treatment.

Table. I. Pill Box frequencies and Q's

\begin{tabular}{|c|c|c|c|c|c|c|}
\hline$\mu^{\prime \prime}$ & $f_{010}[\mathrm{MHz}]$ & $Q_{010}$ & $G_{010} / R_{\mathrm{SQ}}$ & $f_{020}[\mathrm{GHz}]$ & $Q_{020}$ & $G_{020} / R_{\mathrm{SQ}}$ \\
\hline 0 & 883.7 & & & 1.975 & & \\
\hline 1 & 883.0 & 11.03 & 2.3 & 1.968 & 7.2 & 6.5 \\
\hline 2 & 880.9 & 5.42 & 2.6 & 1.924 & 3.2 & 6.2 \\
\hline 3 & 876.5 & 3.51 & 2.5 & 1.772 & 2.0 & 5.7 \\
\hline 4 & 867.8 & 2.52 & 2.3 & 1.603 & 1.7 & 5.3 \\
\hline 5 & 851.0 & 1.90 & 2.2 & 1.479 & 1.7 & 5.0 \\
\hline 6 & 820.6 & 1.49 & 2.0 & & & \\
\hline 7 & 776.9 & 1.23 & 1.9 & & & \\
\hline 8 & 728.6 & 1.07 & 1.8 & & & \\
\hline
\end{tabular}

The frequency and Q-values of the prototype absorber was measured via standard network analyzer methods. The data are interpreted by fitting the TM010 resonance curves with the simulation model in view of deriving the ferrite parameters. Fitting by trial and error is complicated by the availability of the three free parameters, $\mu^{\prime}, \mu^{\prime \prime}, \varepsilon$. The fitting is considerably simplified by establishing the sensitivity of frequency and $\mathrm{Q}$ to parameter changes obtained from the theoretical relations. Fig. 11A shows the frequency changes with $\mu^{\prime \prime}$ while keeping $\mu^{\prime}$ constant. The effect of $\varepsilon^{\prime}$ is negligible in the range considered here. Fig. 11B shows the dependence of $\mu^{\prime \prime}$, which is here insensitive to $\mu^{\prime}$ and $\varepsilon^{\prime}$, a fact also apparent in the computer simulation programs. 

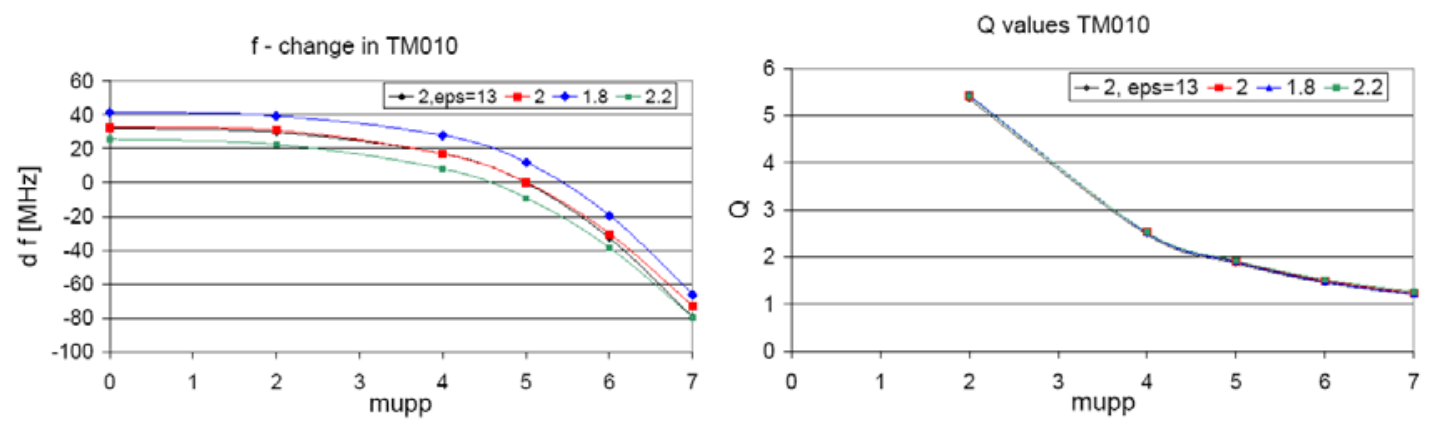

Figure 11. Theoretical dependence of frequency and Q on $\mu^{\prime \prime}$ with $\mu^{\prime}, \varepsilon$ fixed

The analytical model of the ferrite lined pill box provides the electric and magnetic field shape in addition to resonance frequency and quality factor. Comparing field shapes obtained from the various method will be of particular benefit in the evaluating their utility. Using the analytical expressions, the magnetic field shape within the pill box cavity is obtained in the TM010 and TM020 modes for which the ferrite properties are taken as $\mu=2+j 5$ and $\mu=2+j 2$ respectively.

For the TM010 resonance, with $\mu=2+j 5$, the wave numbers are found to be $k_{r i}=(17.84+\mathrm{j} 4.685) / \mathrm{m}, k_{r o}=(139.84-\mathrm{j} 49.20) / \mathrm{m}$. The resonance frequency is 851 $\mathrm{MHz}$ and the quality factor is $Q=\operatorname{Re} k_{r i} / 2 \operatorname{Im} k_{r i}=1.90$. The absolute value of the magnetic field, $H_{\Theta}$, and its real (blue) and imaginary (red) components are shown in Figs. 12. Note the increase of $\left|H_{\Theta}\right|$ with radius in the ferrite region, $r=0.119$ to $0.124 \mathrm{~m}$. Also shown are the corresponding electric field values, with the expected rapid decrease of $\left|E_{z}\right|$ in the ferrite.

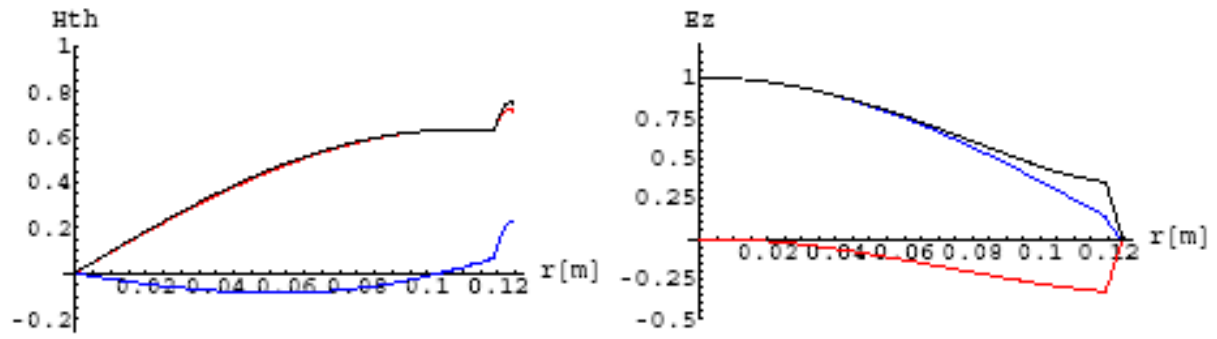

Figure 12. Absolute (black), real (blue) and imaginary (red) component of $H_{\Theta}$ and $E_{z}$

For the TM020 resonance, with $\mu=2-\mathrm{j} 2$, the wave numbers are $k_{r i}=(40.33+\mathrm{j}$ $6.348) / \mathrm{m}$ and $k_{r o}=(231.2-\mathrm{j} 55.75) / \mathrm{m}$. The resonance frequency is $1.924 \mathrm{GHz}$ and the $Q=3.2$. The absolute value of the azimuthal magnet field, $H_{\Theta}$, is shown in Figs. 10 . Note the increase of $\left|H_{\Theta}\right|$ with radius in the ferrite region. Also shown is the absolute value, as well as the real (red) and imaginary (green) component of the axial electric field with its expected rapid decrease in the ferrite. 

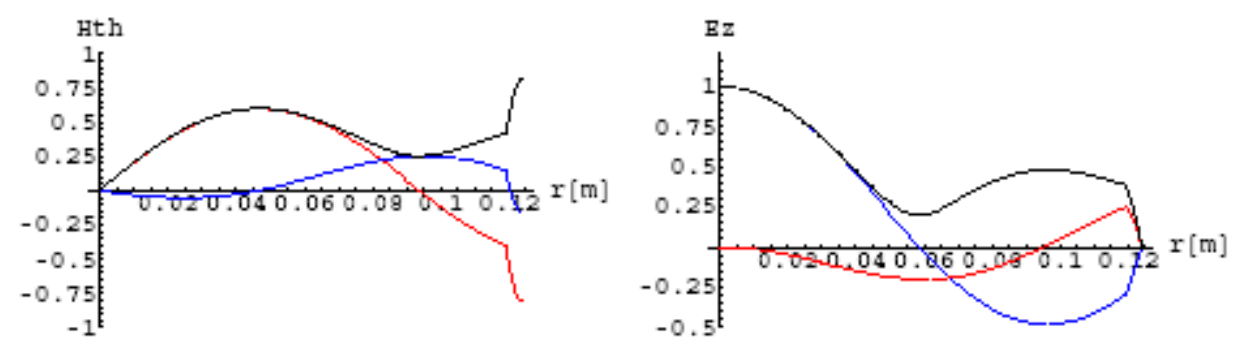

Figures 13. Field strengths of $H_{\Theta}$ (left) and $E_{z}$ (right) in the TM02 mode

The work for the present paper was in part stimulated by the observation of magnetic field plots in the ferrite which differed from the expected z-dependence of all field components in the "unbound" skin effect treatment, which shows a uniformly decaying field into the ferrite. In order to eliminate the suspicion of errors in the simulation codes, the magnetic field shape in the ferrite was computed for the two resonances in the pill box cavity. The absolute value of the magnetic field from the circular analytical and the flat skin effect expressions are shown left and right in Figs.14. The red curves are for the TM010 and the blue for the TM020 mode, with the solid color for no loss and the faded color with losses. The field shape changes from zero $\mu^{\prime \prime}$ to a finite value but the change is relatively weak and no pattern can be detected. The graphs indicate better than qualitative agreement and again confirm the impact of a finite ferrite thickness.
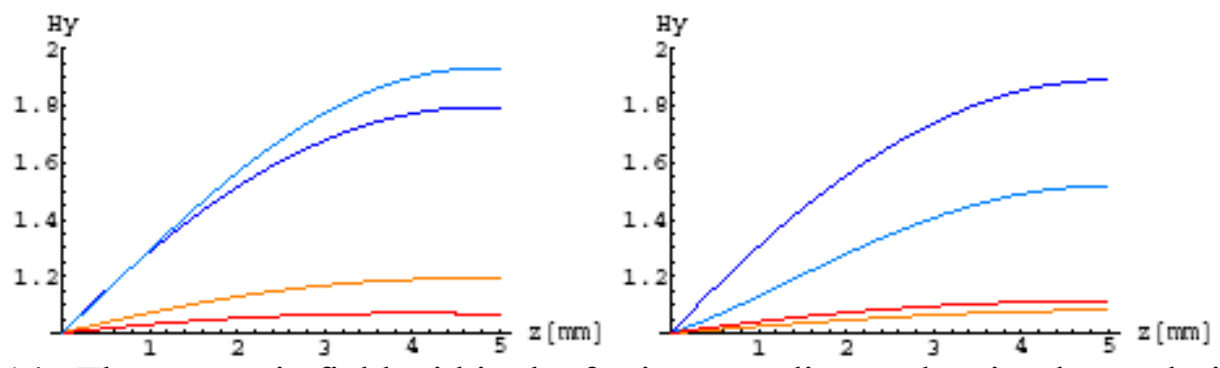

Figures 14. The magnetic field within the ferrite according to the circular analytical (left) and the skin depth analysis (right).

The magnetic field penetrates the ferrite fully and the absorber losses depend on the ferrite thickness. The dependence of the Quality factor in the TM010 mode on the thickness, $\tau$, is plotted in Fig. 15. The results indicate that an increase of the wall thickness beyond the $\sim 3 \mathrm{~mm}$ presently used provides only a small gain and is not cost effective. 


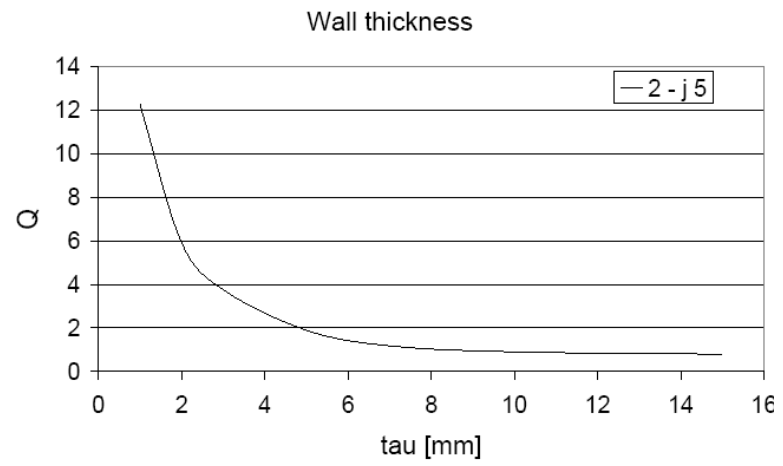

Figure 15. Q-factor dependence on wall thickness

\section{Results from the Micro Wave Studio simulation (L.H \& H.H.)}

The electromagnetic field simulation software, Microwave Studio (MWS) [6], is a threedimensional program to determine various cavity problems in the presence of lossy ferrite and dielectric materials. Applying the code to the ferrite lined pill box represents a convenient test of its accuracy when compared with the analytical results. The program was run for the pill box with a geometry identical to that for the analytical results, that is a $12.4 \mathrm{~cm}$ diameter perfect metallic spool, and a $5 \mathrm{~mm}$ thick and $6.45 \mathrm{~cm}$ long ferrite ring. Several runs were done with different combinations of the imaginary permeability $\mu^{\prime \prime}$, while keeping $\mu^{\prime}=2$ and $\varepsilon^{\prime}=12$ constant. The ferrite parameters are entered as frequency independent $\mu^{\prime}$ and $\mu^{\prime \prime}$ values.

The code is run on a local PC and memory size and time constraints effectively limit the mesh size. Using the largest possible mesh number, $<700,00$, the MWS results for the TM010 resonance frequency and quality factor of the ferrite lined pill box are presented in the Table II and compared with the Mathematica ("m52") solutions.

Table II. MWS Pill Box results

\begin{tabular}{|c|c|c|c|c|}
\hline$\mu^{\prime \prime}$ & $f_{M w S}[\mathrm{MHz}]$ & $f_{m 52}[\mathrm{MHz}]$ & $Q_{M w S}$ & $Q_{m 52}$ \\
\hline 0 & 883.657 & 883.671 & - & - \\
\hline 0.01 & 886.9 & 883.671 & 1100 & 1107.64 \\
\hline 2 & 884.1 & 880.915 & 5.38 & 5.42 \\
\hline 5 & 853.8 & 851.01 & 1.89 & 1.90 \\
\hline
\end{tabular}

The dependence of the simulation results on mesh number is seen in Fig. 16. In the case of maximum mesh size, one has only 5 to 6 radial mesh points in the ferrite region, corresponding to $\sim 1 \mathrm{~mm}$ mesh size, but convergence is sufficient to compare with the analytical data. The resonance frequencies of the lossless cavity from the MWS simulation agree perfectly. The presence of losses slows the convergence speed and leads to divergent final frequencies values. Furthermore it appears here that convergence is faster and comes closer to the exact Q-value, but perhaps only because of the traditionally lower expectations as to the accuracy of the quality factor. The results presented here are obtained in the program by a solver which is based on finding the eigenvalue of a homogeneous matrix. The solution is obtained in the loss-free case via a 
Krylov-Subspace method. For lossy problems, a Jacobi-Davidson solver is used. Using different methods explains the divergent results, but the theoretical Mathematica solution provides the correct frequency values and suggests replacing the Jacobi-Davidson method. The field shape of electric and magnetic fields, not shown here, were compared with those in the above figures and full agreement, at least at the visual level, was found.

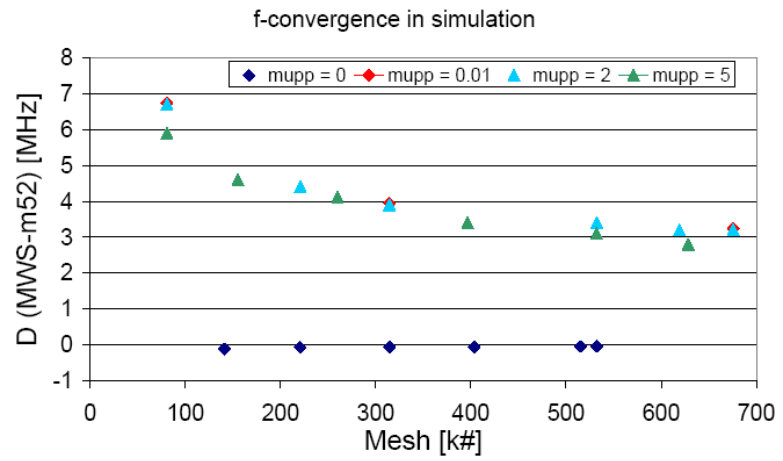

Figure 16. Convergence of MWS frequencies with mesh number.

\section{Results from the GdfidL simulation (A.B.)}

The electromagnetic field solver GdfidL [7] can be used to determine various cavity problems in the presence of lossy ferrite and dielectric materials. Applying the code to the ferrite lined pill box represents a convenient test of its accuracy when compared with the analytical results. The program was run for the pill box with an identical geometry of $12.4 \mathrm{~cm}$ diameter of the perfect metallic box, $5 \mathrm{~mm}$ thick and $6.45 \mathrm{~cm}$ long ferrite ring. The mesh size was chosen as $0.5 \mathrm{~mm}$, leading to 10 radial mesh points in the ferrite.

Several (day-long) runs were done with different combinations of $\mu^{\prime}$ and $\mu^{\prime \prime}$ while keeping $\mu^{\prime}=2$ and $\varepsilon^{\prime}=12$ constant. The resonance frequencies of the empty cavity were found to be $925.7 \mathrm{MHz}$ for TM010 and $2.125 \mathrm{GHz}$ for the TM020 mode (versus 918.6 $\mathrm{MHz}$ and $2.109 \mathrm{GHz}$ from the analytical model).

Ferrite losses are entered into the program as "constant" magnetic conductivity, $\sigma_{\mu}=\mu_{0} \mu^{\prime \prime} \omega$ at $1 \mathrm{GHz}$, which remains constant for all frequencies, but implies a change according to $\mu^{\prime \prime} \sim 1 / f$. The GdfidL results for resonance frequency and quality factor of the ferrite lined pill box are presented in Table III, with $\mu^{\prime}=2$ and $\varepsilon^{\prime}=12$ constant.

Table III. Pill Box results from GdfidL

\begin{tabular}{|l|l|l|l|l|l|c|}
\hline $\begin{array}{l}\sigma_{\mu} \\
{[\Omega / \mathrm{m}]}\end{array}$ & $\mu^{\prime \prime}$ & $f_{G}[\mathrm{MHz}]$ & $Q_{G}$ & $\mu^{\prime \prime}$ & $f_{G}[\mathrm{GHz}]$ & $Q_{G}$ \\
\hline & & TM010 & & & TM020 & \\
\hline 0 & 0 & 884.7 & - & 0 & 1.978 & - \\
\hline 15783 & 2.24 & 891.0 & 4.95 & 1.0 & 1.981 & 7.38 \\
\hline 39457 & 5.40 & 925.4 & 2.1 & 2.49 & 2.007 & 2.5 \\
\hline
\end{tabular}


The electric and magnetic field shape was obtained for the TM010 and TM020 modes resonances with parameters equal to those in Figs. 12 and 13. The TM010 mode in Fig. 17 with $\mu=2-j 5.4$ is resonant at $925.4 \mathrm{MHz}$ with a $Q$ of 2.1 (which is to be compared to $840.7 \mathrm{MHz}$ and 1.9 for the analytical result). Although the field shapes are in good agreement, the numerical frequency differences are significant and are not yet explained. The difference can be attributed in part to the mesh versus continuous treatment, but more likely to the matrix eigenvalue solver.
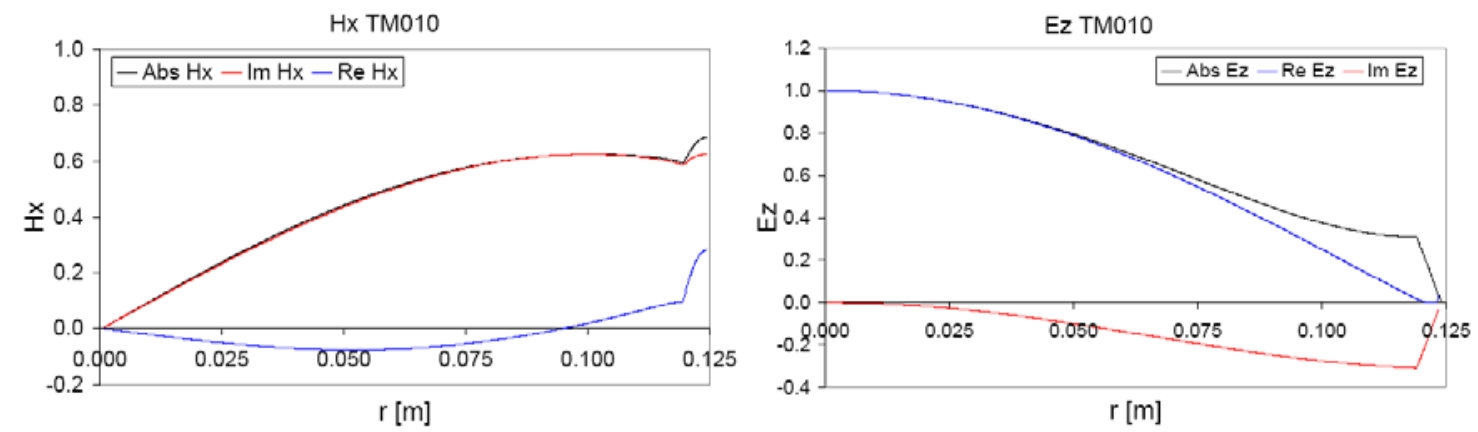

Figure 17. Magnetic and electric field components of the TM010 mode

The TM020 mode in Fig. 14. with $\mu=2-2.49 j$ is resonant at $2.007 \mathrm{GHz}$ with a $Q$ of 2.5 (which is to be compared to $1.861 \mathrm{GHz}$ and 2.41 for the analytical result). Although the field shapes are in good agreement, the numerical differences are significant.
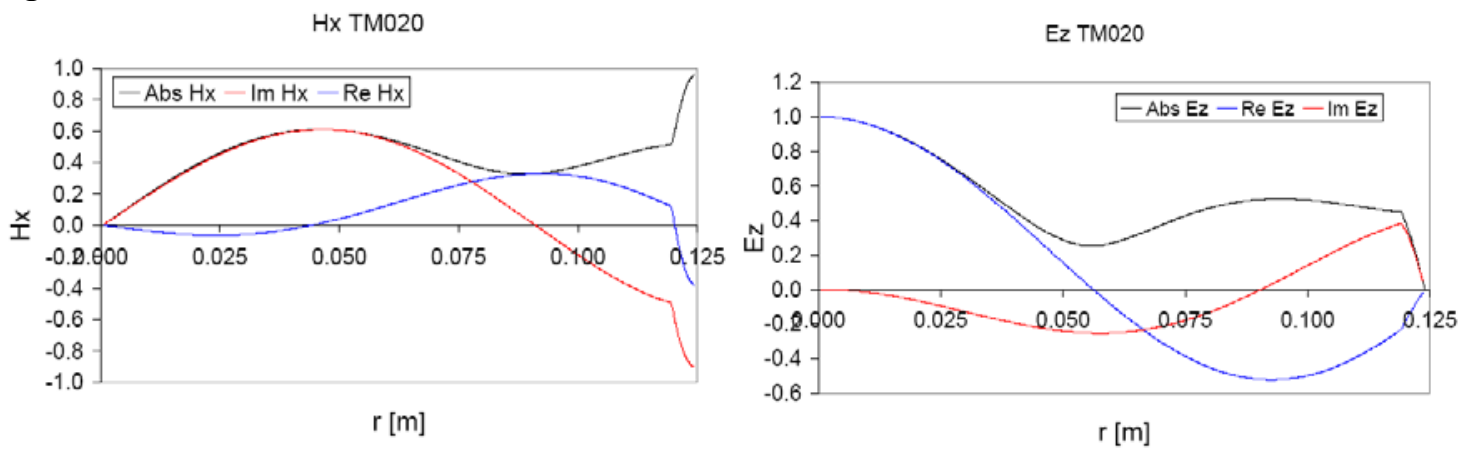

Figure18. Magnetic and electric field components of the TM020 mode

\section{Results from the SUPERFISH simulation (D.K. \& J.R)}

The simulation program Superfish (SFISH)can be conveniently used for finding the eigenmodes of cylindrically symmetric cavities. It accepts complex material parameters, yields resonance frequency, quality factor and field shape. As a result of the geometry constraints, it runs faster than MWS or GdfidL. The SFISH program was run for the pill box cavity with the reference geometry of $12.4 \mathrm{~cm}$ diameter of the perfect metallic pill box and $5 \mathrm{~mm}$ thick long ferrite ring. The mesh size was chosen as $0.5 \mathrm{~mm}$, leading to 10 radial mesh points in the ferrite. The ferrite parameters are entered as $\varepsilon=12, \mu^{\prime}=2$, and frequency independent $\mu^{\prime \prime}=0,2,5$. 
The program requires a starting "driving" point, at which the magnetic field is set to $H_{1}=1$, but no external driving source is implied. Output, especially the resonance frequency depends strongly on the choice of the starting point location. This effect has been explored by running with $H_{1}$ at the center of the cavity with $r=10$ and $12.4 \mathrm{~cm}$, the latter at the outer edge of the ferrite. Furthermore, since resonance frequency and quality factor of the TM010 mode are independent of the pill box length, runs were executed for 6.5 "short" and $65 \mathrm{~cm}$ "long" cavities.

Superfish results are compared in Fig. 19 which shows the dependence of the resonance frequency on the initial point. The TM010 resonance is found by SFISH, stating at $r=10 \mathrm{~cm}$, for the loss-free case to be equal to the theoretical value, 883.7 $\mathrm{MHz}$, but differs strongly in the presence of losses, 753.9 versus the $850.0 \mathrm{MHz}$ for $\mu^{\prime \prime}=5$. The Q-values are essentially independent of the starting point, but show significant differences with theory, 2.62 versus the theoretical 1.90 for $\mu^{\prime \prime}=5$. Due to its relatively short turn around times, SFISH is the work horse for cavity design, but improvements to its eigenmode solver subroutine are necessary for its application to ferrite absorbers.
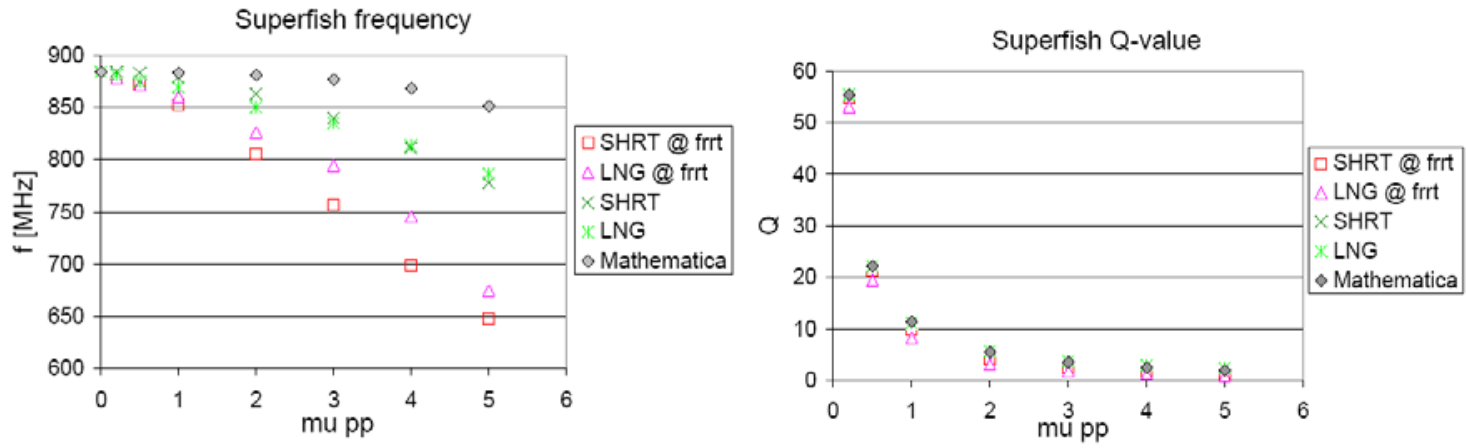

Figure 19. Frequency and Q-value comparison of SFISH with Mathematica

\section{Illustrative Example}

Surplus ferrite tiles were assembled into a very short prototype cavity, with $12.4 \mathrm{~cm}$ diameter and $6.5 \mathrm{~cm}$ length. The cavity had axially located probes to excite rotationally symmetric TM modes when measured with a network analyzer. The S21 transmission coefficient is shown in Fig. 20 . The resonances in the empty cavity at $0.925,2.122$, and $2.492 \mathrm{GHz}$ are interpreted as TM010, TM020, and TM011 respectively.

The TM010 resonance in the ferrite lined cavity is expanded in Fig. 21 and fitted by a resonance at $800 \mathrm{MHz}$ with a $Q \approx 3.6$. Taking the nominal ferrite thickness of 3.175 $\mathrm{mm}$, the Mathematica program provides the permeability of $\mu^{\prime}=5.6$ and $\mu^{\prime \prime}=5.0$. The permeability estimates are, of course, strongly dependent on the ferrite thickness taken: e.g. $5 \mathrm{~mm}$ leads to $\mu^{\prime}=3.8$ and $\mu^{\prime \prime}=3.0$. In contrast, the perturbative interpretation which yields a $R_{S Q}=1.202 \times Z_{0} / Q \approx 130 \Omega$ at $800 \mathrm{MHz}$, is independent of the ferrite thickness assumption and could be used directly in a simulation program. However, the measured $R_{S Q}$ does not lead to the permeability values. 


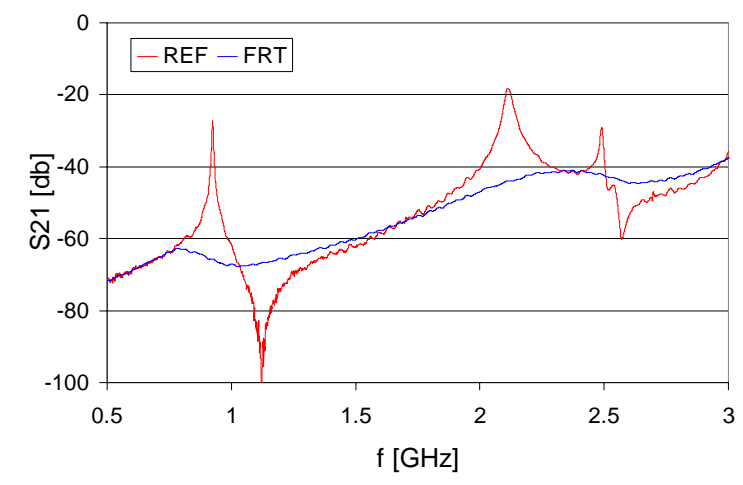

Figure 20. S21 Measurement of short cavity, empty and with ferrite

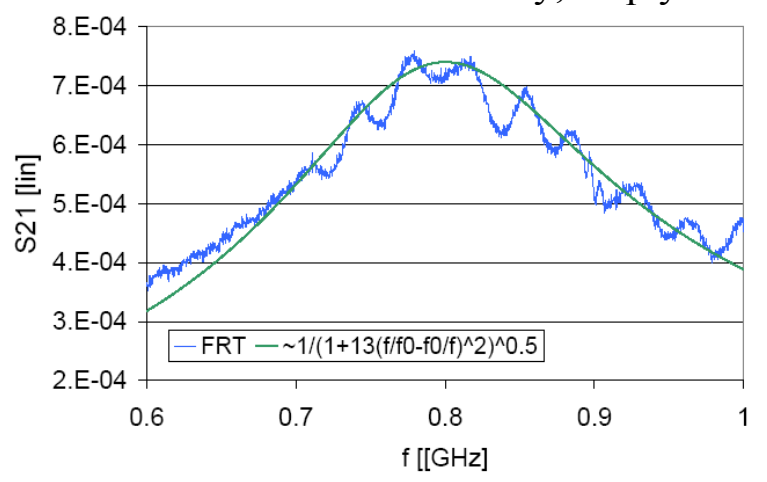

Figure 21. S21 for TM010 resonance direct (blue) and fitted (green).

\section{Discussion}

The HOM damping properties of the ferrite absorber are determined by the material properties, primarily given by the permeability $\mu^{\prime}$ and $\mu^{\prime \prime}$. A limited number of permeability measurements on small samples are available in the pertinent literature, but it seems important to collect performance data for ferrite absorbers in the operational configuration. With this goal in mind, a full-size absorber model was arranged as a ferrite lined pillbox for S21 transfer coefficient measurements with a network analyzer. The cavity is sufficiently small to be only excited at a limited number of resonances, primarily in the TM010 and the TM020 modes, for which frequency and Q-value are found. The present study was initiated to support the interpretation of the results and produce transportable permeability parameters for application to other structures.

In a first exercise, the ferrite losses were interpreted as a perturbation for which the complete skin effect expressions were developed in this paper. Order of magnitude estimates for the ferrite losses, both in the TM010 and the TM020 resonance, were obtained by using the skin effect surface impedance value, together with the geometry factors of the empty cavity. Inversely, simple pillbox cavity Q-measurements can be interpreted as surface impedance and can then serve as guide to the damping properties in ferrite HOM absorbers. The results lead to the conclusion that the measured "skin effect" surface impedance, the $R_{S Q}$ can be used as qualitative guide in estimating losses, but that the ferrite absorber properties in general require a non-perturbative treatment. 
Alternatively, the measured pillbox frequency and Q-value can be used to extract the ferrite parameters by fitting them with results from the theoretical expressions, using $\mu^{\prime}$ and $\mu^{\prime \prime}$ as free parameters. The theoretical field expressions for the ferrite-lined pill box are in principle rigorous and without much effort provide resonance frequencies and Q-values via the Mathematica program. This procedure has the advantage of eliminating the need to know the actual geometry constant.

A general procedure, discussed in this paper and suitable to a cavity without geometrical constraints, consists in fitting the measured S21 transfer coefficient with curves computed by a simulation program such as Microwave Studio, GdifidL, and SuperFish. The accuracy of simulation programs is dependent on the mesh construction and the eigenvalue solvers employed. An important objective of the present study thus was establishing confidence in the performance of simulation programs. This was attempted by comparing the simulated with the exact theoretical frequency and Q-values of a ferrite lined pillbox with prescribed permeability. Although all simulations produced full agreement with theory for the loss-free case, only the Microwave Studio gave qualitatively correct results for lossy structures. The impact of a limited accuracy on the interpretation remains to be examined, but the present study suggests the need for improvement of the simulation programs.

\section{Acknowledgements}

The authors would like to thank Drs. V. Litvinenko and I. Ben-Zvi for their comments and illuminating discussions.

\section{References}

1. Ilan Ben-Zvi, Proc. 2005 PAC, Knoxville, TN, p. 2751.

2. T. Tajima, et al., Proc PAC 1999, New York, NY, p. 440

3. S. Belomestnykh, et al., Proc PAC 1999, New York, NY, p.980

4. E. Chojnacki and W. J. Alton, Proc. 1999, New York, NY, p.845

5. H. Hahn et al., Physica C, 239 (2006)

6. CST-Computer Simulation Technology, Darmstadt, Germany

7. W. Bruns, Electromagnetic Field Solver,GdfidL, http://www.gdfidl.de/; Proc. LINAC 2002, Gyeongju, Korea, p. 416.

8. W. H. Hartung, Proc. 1993 PAC, Dallas, TX, p.3450, and The Interaction between a Beam and a Layer of Microwave-Absorbing Material, (Dissertation, Cornell University, 1966)

9. J. Mouris and R. M. Hutcheon, Measurements of the complex microwave permeability of un-biased Ferrite C-48 and Ferrite-50, from room temperature to $200{ }^{\circ} \mathrm{C}$ at frequencies between $915 \mathrm{MHz}$ and $2800 \mathrm{MHz}$., Report MPN-41-00 (Canadian Light Source / Microwave Properties North, December 2000)

10. V. Shemelin et al., NIM in Physics Research, A557,p. 268 (2006)

11. N. Marcuvitz, ed., Waveguide Handbook, (McGraw Hill Book Co, New York, 1951), p.67 